\title{
VARIACIÓN TEMPORAL DE LA EROSIÓN POR CÁRCAVAS EN LOS FONDOS DE VALLE BAJO EXPLOTACIÓN DE DEHESA
}

\author{
A. GÓMEZ GUTIÉRREZ ${ }^{(1)}$, S. SCHNABEL ${ }^{(1)}$ \\ y J. J. SANJOSÉ BLASCO ${ }^{(2)}$ \\ (1) Grupo de Investigación GeoAmbiental, \\ (2) Grupo de Ingeniería Geomática, \\ Universidad de Extremadura. Avda. de la Universidad, 10071 Cáceres \\ Correo electrónico de contacto: alvgo@unex.es
}

\begin{abstract}
RESUMEN. En este trabajo se analiza la erosión en cárcavas de fondo de valle en las dehesas del SO de España y su relación con la hidrología y el uso del suelo. El estudio se desarrolló en Parapuños, una pequeña cuenca con una superficie de 99,5 ha. Se utilizaron dos metodologías diferentes: i) en primer lugar, se determinó la erosión en cárcava a través del levantamiento topográfico de perfiles transversales a lo largo del cauce, mientras se registraban automáticamente el caudal y la precipitación para el período 2001-2006, ii) en segundo lugar, se analizó la evolución de la cárcava y el uso del suelo a partir de cartografía elaborada con ortofotografías de diferentes fechas para el período 1945 2006. Las pérdidas registradas a partir de la medición de los perfiles topográficos, presentan una alta variabilidad temporal. Pese a que los datos indican la existencia de una relación entre caudal y acarcavamiento, es posible que esta no sea tan simple. La presencia de diferentes procesos de erosión en el cauce añade aún más complejidad, con grandes variaciones espaciales y estacionales, indicando la importancia del contenido de agua en las paredes de la cárcava y la disponibilidad de sedimentos para ser removidos. Respecto a los resultados obtenidos a partir de las ortofotografías aéreas, el área afectada aumentó de $695 \mathrm{~m}^{2}$ en 1945 a $1009 \mathrm{~m}^{2}$ en 2006, alcanzando un máximo de $1560 \mathrm{~m}^{2}$ en 1956. Esta área acarcavada aparece fuertemente relacionada con el uso del suelo, especialmente con la superficie cultivada dentro de la cuenca, siguiendo ambas variables una evolución similar durante el período de estudio.
\end{abstract}

ABSTRACT. This paper analyzes gully erosion in valley bottoms of rangelands with a disperse tree cover in SW Spain (dehesas) and its relation with hydrology and land use. The study was carried out in a small catchment (99.5 ha). Two different approaches were used; i) first, gullying was determined by means of a repeated survey of topographic cross sections and discharge and rainfall were 
monitored continuously since 2001, ii) secondly, the evolution of the gully and land use was analyzed using maps elaborated from aerial orthophotographs for the period from 1945 to 2006. Sediment losses present strong temporal variation. Although data indicate a relation between discharge and gullying, no simple relationship exists. Different erosion processes operating in the channel add to the complexity, varying in space and seasonally, indicating the importance of the water content of the gully banks and sediment availability. Regarding the area affected by gullying, the analysis of the aerial orthophotographs shows an increase from $695 \mathrm{~m}^{2}$ in 1945 to $1009 \mathrm{~m}^{2}$ in 2006, reaching a maximum of 1560 $m^{2}$ in 1956. This gullied area is closely related with land use, especially with the amount of cultivated areas within the catchment, with a similar evolution during the study period.

Palabras clave: erosión por cárcavas, caudal, precipitación, uso del suelo, dehesas.

Key words: gully erosion, discharge, rainfall, land use, rangelands.

Enviado el 15 de septiembre de 2008 Aceptado el 27 de enero de 2009

\section{Introducción}

Las cárcavas de fondo de valle son un fenómeno bastante frecuente en áreas semiáridas con pastoreo extensivo. Aunque se encuentran asociadas a las líneas de drenaje y su generación se vincula con los flujos superficiales, la información acerca de su relación con la escorrentía es escasa (Crouch, 1990; Thomas et al., 2004). Este hecho puede ser debido, en parte, a la elevada variabilidad temporal del acarcavamiento que exige un seguimiento prolongado de caudal y erosión. Los factores que determinan el proceso de acarcavamiento son muy variados, pero se pueden agrupar en cuatro tipos: edáfico-litológicos, climáticos, topográficos y de uso y manejo. Entre ellos, los factores relacionados con el clima y el manejo son de especial interés, ya que actualmente presentan una mayor tendencia al cambio a corto y medio plazo.

La erosión a lo largo de una cárcava varía notablemente, produciéndose pérdidas elevadas por el retroceso de cabeceras y por el colapso de sus paredes como consecuencia de incisión lateral (Schnabel, 1997). Los procesos de erosión en cárcava también muestran una elevada variabilidad temporal y están relacionados con los flujos superficiales, ya que no se han encontrado indicios de piping.

El desarrollo o aparición de sistemas de cárcavas también se ha asociado en numerosas ocasiones a cambios en el uso del suelo o en los sistemas de manejo y explotación (Faulkner, 1995; Harvey, 1996; Prosser y Winchester, 1996; Martínez-Casasnovas y Sánchez-Bosch, 2000; Chaplot et al., 2005; Kasai, 2006; Parkner et al., 2006). En esta línea, las dehesas del SO de España son un paisaje especialmente atractivo para el estudio de las relaciones entre los diferentes usos y los procesos de degradación del suelo, ya que 
han experimentado grandes cambios durante el último siglo. El hecho más significativo y que resume la historia reciente de la dehesa, es una transición de un aprovechamiento múltiple y tradicional a otro moderno basado en la ganadería. Dicha transformación, aparece motivada por una crisis de la agricultura tradicional y la adopción de la Política Agraria Común (PAC) debido al ingreso de España en la Unión Europea en 1986.

El objetivo principal de este trabajo es analizar los factores que intervienen en la variación temporal del acarcavamiento a partir de dos metodologías distintas y con un marco temporal de análisis también diferente. Por un lado, se pretende estudiar las relaciones entre la erosión que tiene lugar en la cárcava y la precipitación y el caudal a partir del levantamiento topográfico de perfiles transversales y del registro automatizado de caudal y precipitación en la cuenca para el período 2001-2006. Por otra parte, se pretende analizar la evolución de la cárcava durante los últimos 60 años y su relación con el uso del suelo a partir del análisis de ortofotografías aéreas.

\section{2. Área de estudio}

La cuenca Parapuños se encuentra al NE de la ciudad de Cáceres. Se trata de un área representativa de dehesas, con un relieve ondulado sobre esquistos y grauvacas precámbricas. La vegetación presenta densidades variables de encinas (Quercus rotundifolia) y de arbustos (principalmente Retama sphaerocarpa). El uso actual se basa en el pastoreo extensivo de ganado ovino y porcino. Las características principales de esta cuenca se resumen en la Tabla 1. La cárcava se desarrolla sobre el fondo de valle, en la parte baja de la cuenca, erosionando depósitos aluviales que alcanzan espesores de 1 a $2 \mathrm{~m}$. Se trata de un canal discontinuo con varias cabeceras y anchuras que varían entre 2 y $5 \mathrm{~m}$. La cárcava puede dividirse en tres tramos de cauce; uno principal que se extiende 630 m con un área de drenaje de 49,9 ha y una pendiente media del 7,96\%, un tributario que se extiende $133 \mathrm{~m}$ con un área de drenaje de 45,4 ha y una pendiente media del 8,02\% y finalmente el tramo que discurre desde la confluencia de los dos anteriores hasta la salida de la cuenca que se extiende $174 \mathrm{~m}$ y presenta una pendiente media del 7,91\% y un área de drenaje de 4,2 ha.

Los sedimentos sobre los que se desarrolla la cárcava, presentan una textura dominantemente franco-arenosa con bajos contenidos de arcilla y con contenidos variables de elementos gruesos. Existe una capa relativamente continua donde los elementos gruesos suponen más del $70 \%$ que se sitúa a partir de los $70 \mathrm{~cm}$ de profundidad y generalmente se extiende hasta el contacto con el sustrato (la profundidad de este contacto varía entre $90 \mathrm{~cm}$ y $160 \mathrm{~cm}$ ). En el resto del perfil, los elementos gruesos no superan el 10\%. La densidad aparente es de 1,6 $\mathrm{g} \mathrm{cm}^{-3}$. Son suelos ácidos con un bajo contenido de materia orgánica, que en los primeros centímetros no supera el $3 \%$. Los suelos de las laderas son poco profundos y de textura franco arenosa a limosa. El clima es mediterráneo con un marcado período seco estival, mostrando las precipitaciones una elevada variabilidad interanual. 
Tabla 1. Principales características de la cuenca experimental Parapuños

\begin{tabular}{|l|c|}
\hline Variables & Parapuños \\
\hline Superficie (ha) & 99,5 \\
Orientación & SSO \\
Altitud máxima y mínima (m) & $434-362$ \\
Desnivel absoluto (m) & 72 \\
Pendiente media (\%) & 7,91 \\
Desnivel/longitud cuenca & 0,055 \\
Orden de la cuenca & 2 \\
Longitud de la cárcava (m) & 833 \\
Pendiente de la cárcava (\%) & 2,65 \\
Sustrato & Pizarras y sedimentos (raña) \\
Arbolado (pies/ha) & $0-60$ \\
Ganado & ovino, porcino \\
\hline
\end{tabular}

\section{Metodología}

\subsection{Evolución del acarcavamiento 2001-2006}

La estimación del volumen del material erosionado desde el 2001 hasta el 2006 se basa en la monitorización de 28 perfiles transversales a lo largo del cauce. El levantamiento de estos perfiles se realiza utilizando una estación total láser y con una frecuencia aproximada de 6 meses. El volumen del material erosionado (o depositado) a lo largo de la sección de cauce delimitado por dos perfiles consecutivos para el período transcurrido entre dos levantamientos, se estima como la media del cambio registrado en ambas secciones durante dicho período por la distancia que las separa. La distribución de los perfiles a lo largo del cauce es irregular, y fue definida incrementando la densidad de los mismos en las zonas que denotaban una mayor actividad.

Paralelamente, se han medido caudal y precipitación de forma continua con una resolución temporal de 5 minutos. El caudal se determina con un sensor de profundidad instalado en un aforador en forma de $\mathrm{V}$ y con sección de aproximación trapezoidal, situado a la salida de la cuenca. La precipitación se registra con pluviómetros de balancín tipo Onset RG2-M distribuidos por toda la cuenca. Pese a la realización de un registro continuo de precipitación y caudal, las características de los datos de evolución de la cárcava no permiten la realización de un análisis a escala de evento. 


\subsection{Evolución del acarcavamiento 1945-2006}

El análisis de la evolución de la cárcava desde 1945 hasta 2006 se basó en la realización de cartografía de la misma a partir de ortofotografías aéreas. Sobre los fotogramas aéreos originales se llevó a cabo un proceso de ortorrectificación. Dicho proceso consiste en la modelización matemática de la geometría de la formación de la imagen considerando las diferentes fuentes de error. Las ortofotografías resultantes presentan la peculiaridad de combinar el detalle de visualización de un fotograma aéreo y las características geométricas de un plano. Sobre cada ortofoto, se delimitó el área afectada por la cárcava. Esto es posible debido a que las cárcavas presentan paredes laterales verticales y un límite aguas arriba claramente definido: su cabecera. Al mismo tiempo, los materiales que se localizan sobre la base del lecho (sedimentos, sustrato, suelo desnudo, etc.) suelen diferir de los encontrados en las márgenes. Del mismo modo se delimitaron unidades con cobertura vegetal uniforme sobre cada ortofotografía. Paralelamente, se construyó un catálogo de posibles usos del suelo en la cuenca desde 1945 hasta el 2006 a partir de estudios de campo realizados desde el 2001, entrevistas con testigos históricos y estudios previos realizados por otros autores (Plieninger, 2006; Plieninger y Harald, 2006). Finalmente se asignó un uso o unidad de cubierta vegetal a cada una de las unidades delimitadas sobre la ortofotografía. El resultado final es un mapa del área acarcavada y de unidades de cubierta vegetal y uso del suelo para cada fecha. En la Tabla 2 se pueden observar las características de las fotografías aéreas originales y de las ortofotografías resultantes.

Debido a la diversidad de las características de las fotografías aéreas originales (escala, modo color, película, etc.) se llevó a cabo una evaluación del error cometido al realizar mediciones de longitudes y áreas sobre cada ortofotografía. Dicho análisis consistió en la comparación de los valores de longitud y área obtenidos al digitalizar sobre cada ortofotografía determinados elementos del paisaje con su valor real registrado en campo. El error cuadrático medio obtenido para la medición de distancias fue de $0,75 \mathrm{~m}$ mientras que el error cuadrático medio obtenido para el registro de áreas fue de 4,63 $\mathrm{m}^{2}$.

Tabla 2. Características del material fotográfico utilizado y de las ortofotografías resultantes, incluido el error cuadrático medio para la medición de longitudes $\left(R M S_{L}\right)$ y áreas $\left(R M S_{A}\right)$ sobre las ortofotografías

\begin{tabular}{|l|c|c|c|c|c|}
\hline \multicolumn{1}{|c|}{ Fecha } & $\begin{array}{c}\text { Escala } \\
\text { Fotografía }\end{array}$ & Modo Color & $\begin{array}{c}\text { Tamaño } \\
\text { Píxel (m) }\end{array}$ & $\begin{array}{c}\mathbf{R M S}_{\mathbf{L}} \\
\mathbf{( m )}\end{array}$ & $\begin{array}{c}\mathbf{R M S}_{\mathbf{A}} \\
\mathbf{( m}^{\mathbf{2}} \mathbf{)}\end{array}$ \\
\hline $28-30 / 09 / 1945$ & $1: 44.000-43.000$ & Escala de grises & 0,5 & 1,10 & 15,82 \\
$17 / 04 / 1956$ & $1: 35.000$ & Escala de grises & 0,5 & 0,70 & 3,16 \\
$08 / 1989$ & $1: 20.000$ & Escala de grises & 0,5 & 0,64 & 1,88 \\
$02 / 1998$ & $1: 40.000$ & Escala de grises & 1 & 1,20 & 4,42 \\
$19 / 06 / 2002$ & $1: 6.000$ & RGB & 0,2 & 0,19 & 1,09 \\
$26-29 / 04 / 2006$ & $1: 30.000$ & RGB & 0,5 & 0,64 & 1,44 \\
\hline
\end{tabular}




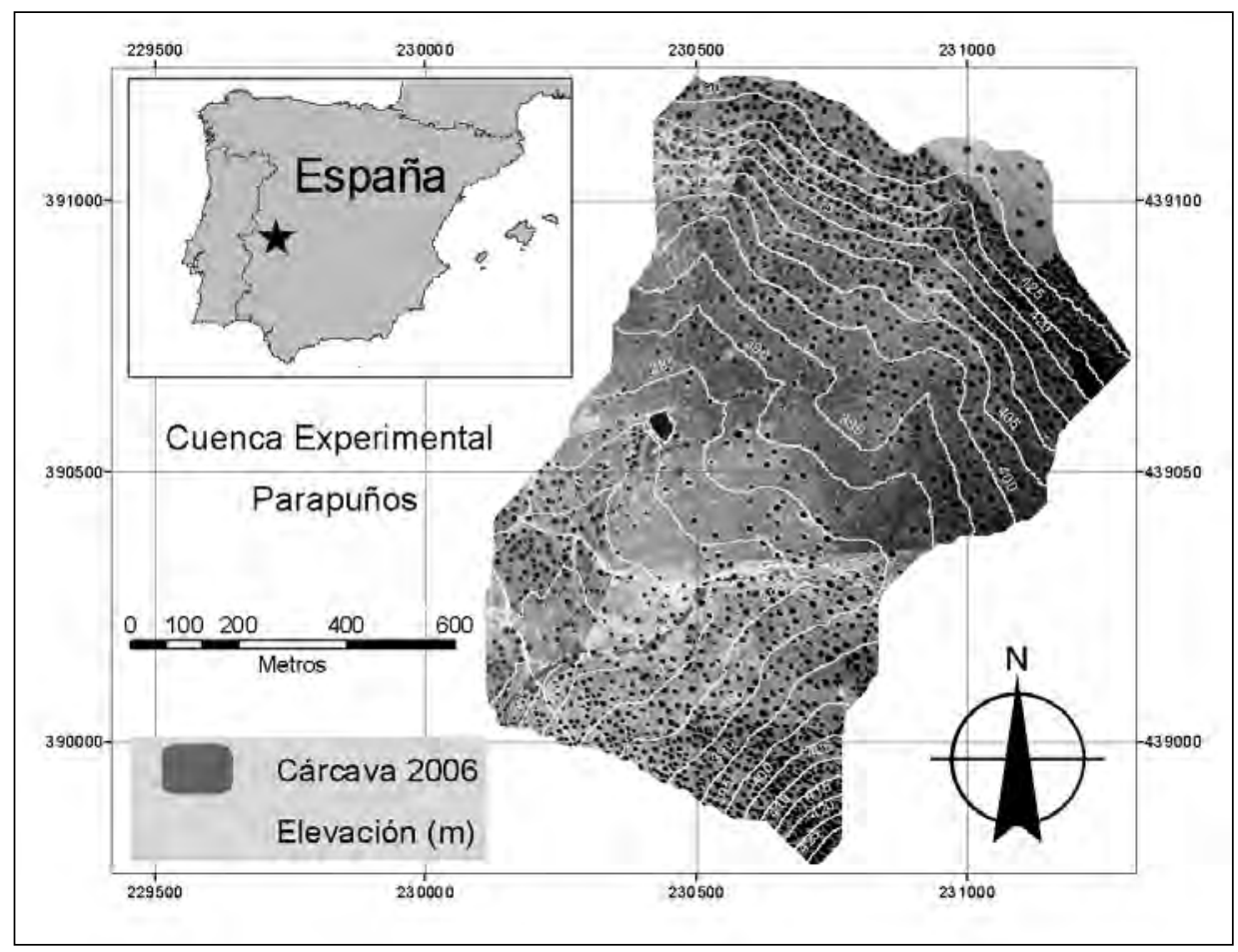

Figura 1. Localización de la cuenca experimental Parapuños, extensión de la cárcava en 2006 sobre ortofotografía y curvas de nivel con equidistancia de $5 \mathrm{~m}$

Para analizar el impacto de la precipitación total durante el período transcurrido entre dos fotografías aéreas sobre el avance de la cárcava, se construyó una base de datos de precipitación a partir de datos directos registrados en la cuenca (desde 2001) y de correlaciones entre los datos de la cuenca y dos estaciones cercanas: Monroy (con datos para el período 1961-1990 y 1999-2006) y Cáceres (con datos para el período 1945-1960 y 1991-1998), situadas a 5,85 km y 25,80 km de Parapuños, respectivamente. El ajuste entre los datos de las tres estaciones para la obtención de la base de datos general resultó en coeficientes de correlación significativos $\left(\mathrm{R}^{2}=0,81\right.$ y $\mathrm{R}^{2}=0,99$ con $\left.\mathrm{p}<0,05\right)$.

\section{Resultados}

\subsection{Evolución del acarcavamiento 2001-2006}

La tasa media de erosión durante este período en la cárcava de Parapuños fue de $-3,15 \mathrm{~m}^{3} \mathrm{a}^{-1}$, alcanzando un máximo de erosión de -18,18 $\mathrm{m}^{3}$ (erosión) en el período transcurrido de julio de 2002 a enero de 2003 (P1; Tabla 3) y un mínimo de 11,07 m³ (deposición) 
Tabla 3. Erosión (valores negativos) y deposición en cárcava en Parapuños. Se presentan los totales para cada período de medición, junto con las cantidades de precipitación, máximas instantáneas de caudal (Qmax), aportación y el número de veces que el caudal de un evento superó $1000 \mathrm{~m}^{3}(Q>1000)$

\begin{tabular}{|c|c|c|c|c|c|c|c|}
\hline $\begin{array}{l}\text { Período } \\
\text { de } \\
\text { medición }\end{array}$ & Período & $\begin{array}{c}\text { Duración } \\
\text { n meses }\end{array}$ & $\begin{array}{c}\text { Lluvia } \\
(\mathrm{mm})\end{array}$ & $\begin{array}{c}\text { Erosión/ } \\
\text { Deposición } \\
\left(\mathbf{m}^{3}\right)\end{array}$ & $\begin{array}{c}\text { Qmax } \\
\left(\mathrm{m}^{3} \mathbf{k m}^{-2} \mathbf{s}^{-1}\right)\end{array}$ & $\begin{array}{c}\text { Aportación } \\
\left(\mathbf{m}^{\mathbf{3}}\right)\end{array}$ & $\begin{array}{c}Q>1000 \\
\text { (n) }\end{array}$ \\
\hline $\begin{array}{l}\text { Dic } 2001 \\
- \text { Jul } 2002\end{array}$ & P1 & 7 & 247,5 & $-1,49$ & 0,46 & $3.436,6$ & 1 \\
\hline $\begin{array}{l}\text { Jul } 2002 \\
\text { - Ene } 2003\end{array}$ & $\mathrm{P} 2$ & 6 & 410,5 & $-18,18$ & 0,46 & $58.164,8$ & 11 \\
\hline $\begin{array}{l}\text { Ene } 2003 \\
\text { - Jun } 2003\end{array}$ & P3 & 6 & 168,3 & 9,42 & 0,30 & $15.355,0$ & 4 \\
\hline $\begin{array}{l}\text { Jun } 2003 \\
\text { - Ene } 2004\end{array}$ & P4 & 6 & 296,2 & $-15,16$ & 1,16 & $22.556,6$ & 6 \\
\hline $\begin{array}{l}\text { Ene } 2004 \\
\text { - Jul } 2004\end{array}$ & P5 & 6 & 212,3 & $-5,02$ & 0,33 & $16.035,6$ & 4 \\
\hline $\begin{array}{l}\text { Jul } 2004 \\
\text { - Ene } 2005\end{array}$ & P6 & 6 & 259,1 & 1,46 & 1,12 & $26.437,8$ & 6 \\
\hline $\begin{array}{l}\text { Ene } 2005 \\
\text { - Jul } 2005\end{array}$ & P7 & 6 & 81,4 & 11,07 & 0,00 & 12,8 & 0 \\
\hline $\begin{array}{l}\text { Jul } 2005 \\
\text { - Dic } 2005\end{array}$ & P8 & 6 & 212,3 & 0,74 & 1,59 & $16.875,1$ & 2 \\
\hline $\begin{array}{l}\text { Dic } 2005 \\
\text { - Jun } 2006\end{array}$ & P9 & 6 & 208,8 & 2,72 & 0,27 & $8.073,1$ & 1 \\
\hline
\end{tabular}

en el período de enero de 2005 a julio del mismo año (P2; Tabla 3). Durante el P4 también se registraron elevadas pérdidas de material en la cárcava $\left(-15,16 \mathrm{~m}^{3}\right)$. Estos períodos con elevadas pérdidas sucedieron durante el primer semestre de sus respectivos años hidrológicos. Por otra parte, los períodos en los que se registró una mayor deposición, tuvieron lugar durante el segundo semestre de sus años hidrológicos: P3, P7 y P9. En la Tabla 3 se pueden observar las características de cada período (duración, erosión/deposición, precipitación y caudales).

Con el propósito de profundizar en las relaciones precipitación-caudal-erosión en cárcava, se llevó a cabo un análisis de correlación, cuyos resultados se presentan en la Tabla 4. A partir de estos resultados se observa una relación significativa entre la erosión/deposición y la aportación con un coeficiente $\mathrm{r}$ de -0,74. La deposición de materiales domina con aportaciones inferiores a $\operatorname{los} 18.000 \mathrm{~m}^{3}$. También resulta muy significativa la relación entre la cantidad total de precipitación y la erosión/deposición registradas, con un coeficiente $r$ de $-0,90$. Algunos parámetros determinados a partir de las características de los eventos también arrojan relaciones significativas con el acarca- 
Tabla 4. Matriz de correlación de erosión/deposición de los diferentes tramos de la cárcava en Parapuños, precipitación y propiedades de caudal

$\left(Q>1000, Q>100, Q \max >100\right.$ - número de veces que se supera un caudal de $1000 \mathrm{~m}^{3}, 100 \mathrm{~m}^{3}$ y un caudal máximo de $100 l \mathrm{~s}^{-1}$, respectivamente)

\begin{tabular}{|l|c|c|c|c|c|c|}
\hline & $\begin{array}{c}\text { Precipitación } \\
(\mathbf{m m})\end{array}$ & $\begin{array}{c}\mathbf{Q t o t} \\
\left(\mathbf{m}^{\mathbf{3}}\right)\end{array}$ & $\begin{array}{c}\mathbf{Q m a x} \\
\left(\mathbf{I ~ s}^{\mathbf{1}}\right)\end{array}$ & $\begin{array}{c}\mathbf{C} \text { Cárc } \\
\left(\mathbf{m}^{\mathbf{3}}\right)\end{array}$ & $\begin{array}{c}\mathbf{Q > 1 0 0 0} \\
\left(\mathbf{n}^{\mathbf{0}}\right)\end{array}$ & $\begin{array}{c}\mathbf{Q}>\mathbf{1 0 0} \\
\left(\mathbf{n}^{\mathbf{0}}\right)\end{array}$ \\
\hline Qtot & $0,86^{* *}$ & & & & & \\
Qmax & 0,33 & 0,24 & & & & \\
Cárcava completa & $-0,90^{* *}$ & $-0,74^{*}$ & $-0,30$ & & & \\
Q>1000 & $0,88^{* *}$ & $0,89^{* *}$ & 0,01 & $-0,71^{*}$ & & \\
Q>100 & $0,84^{* *}$ & $0,96 * *$ & 0,21 & $-0,75^{*}$ & $0,87 * *$ & \\
Qmax>100 & $0,86^{* *}$ & $0,93^{* *}$ & 0,20 & $-0,76^{*}$ & $0,89 * *$ & $0,98^{* *}$ \\
\hline
\end{tabular}

$* *, *$ significativas a $\mathrm{p}<0,001$ y $0,050, \mathrm{~N}=9$

vamiento, como el número de veces que los máximos de crecida superan un caudal de $1001 \mathrm{~s}^{-1}$, el número de veces que se producen caudales superiores a $100 \mathrm{~m}^{3}$ y $1.000 \mathrm{~m}^{3}$ con coeficientes $\mathrm{r}$ de $-0,76,-0,75$ y $-0,71$, respectivamente.

En la Figura 2 se presentan los valores de erosión en cárcava obtenidos junto con la distribución temporal de la precipitación y el caudal. En dicho gráfico puede observarse la tendencia a la concentración de las tasas negativas (erosión) en el primer semestre de los años hidrológicos y la de las tasas positivas (deposición) durante el segundo semestre de los años hidrológicos. Durante el período de estudio, en dos ocasiones se produce la sucesión de dos períodos de pérdidas (P1-P2 y P4-P5).

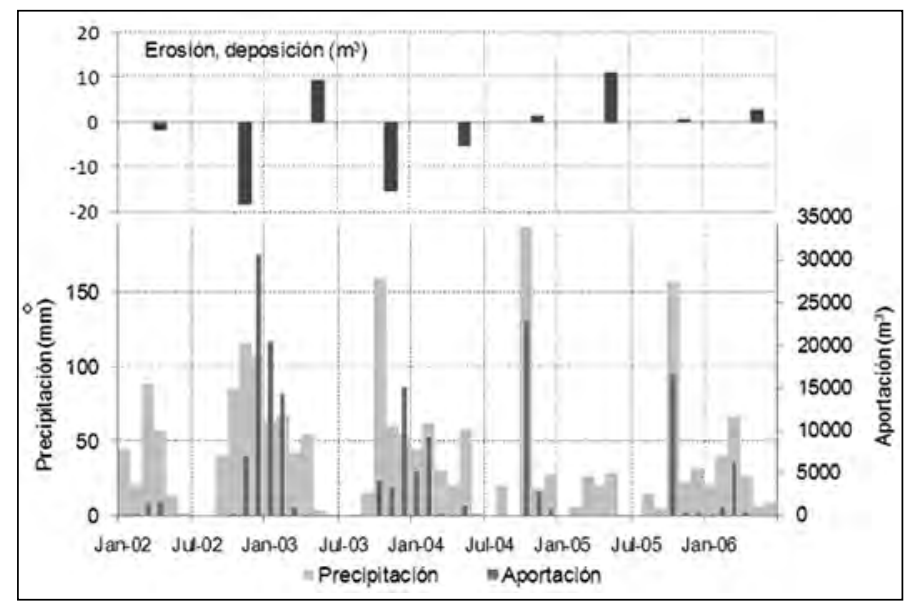

Figura 2. Distribución temporal de la precipitación, el caudal y la erosión/deposición registrada en cada periodo 
Finalmente se ilustran en la Figura 3 las relaciones entre el acarcavamiento y la precipitación y el caudal. Pese al número de datos $(\mathrm{N}=9)$ la distribución de los puntos se localiza a lo largo del rango de valores.
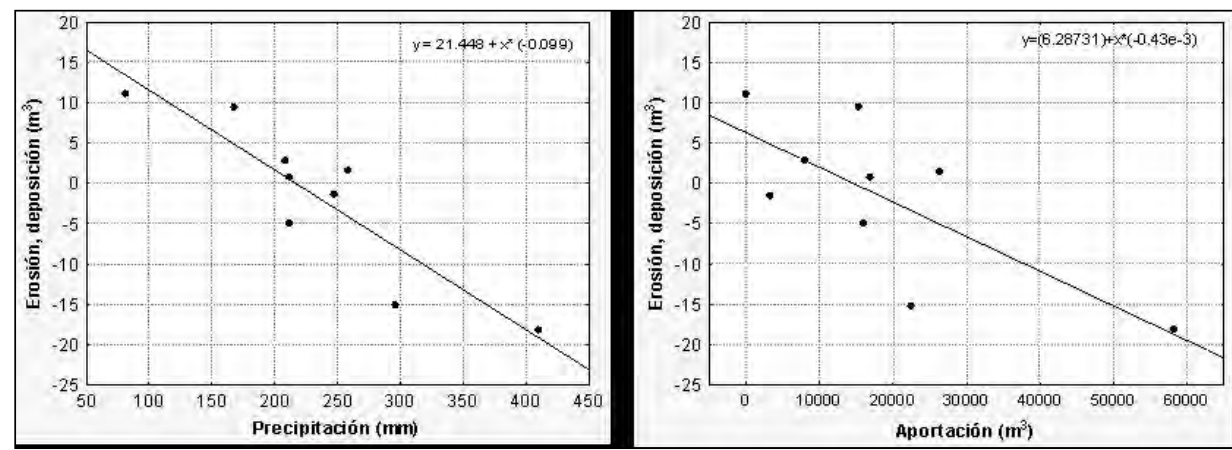

Figura 3. Relación entre la erosión/deposición y la precipitación (izquierda) $(R 2=0,810, p=0,0027)$ y la aportación (derecha) $(R 2=0,541, p=0,0239)$

\subsection{Evolución del acarcavamiento 1945-2006}

La superficie afectada por acarcavamiento experimenta un incremento de 314,10 $\mathrm{m}^{2}$ desde 1945 hasta 2006, lo que supone una tasa de avance de $5,18 \mathrm{~m}^{2} \mathrm{año}^{-1}$. El acarcavamiento alcanza su máximo en 1956 con un área afectada de $1.559,58 \mathrm{~m}^{2}$, de forma que entre 1945 y 1956 se produce una tasa de avance de 82,16 $\mathrm{m}^{2} \mathrm{año}^{-1}$ (Tabla y Tabla). Este período coincide con la puesta en labor de casi la mitad de la superficie de la cuenca, de hecho, el área afectada por acarcavamiento y la superficie cultivada dentro de la cuenca siguen una evolución similar durante el período de estudio (Figura 4)

La evolución del uso del suelo constatada entre los años 1945 y 1956 es similar a la observada por otros autores a escala de municipio en Extremadura (Plieninger, 2006) y se encuentra motivada por una búsqueda del aumento en la productividad de las tierras. En 1945 la totalidad de la superficie de la cuenca se encuentra dedicada a pastoreo o usos forestales, mientras que en 1956 un total de 41,47 ha aparecen ya como zonas de cultivo.

En el período que transcurre desde 1956 hasta 1989 la superficie afectada por acarcavamiento se ve reducida a menos de la mitad (Tabla). Esta tendencia se confirma con los datos de la superficie acarcavada en 1998. Al mismo tiempo, se produce el abandono de la mayor parte de los cultivos debido a la imposibilidad de superar la crisis de la agricultura tradicional. En la cuenca tan sólo se conservan 2,84 ha destinadas al cultivo en 1989 y 1998. Como consecuencia de este abandono, se produce un cambio en la cubierta vegetal de la cuenca; la superficie anteriormente ocupada por cultivos se convierte ahora en pastizales con escasa vegetación leñosa (Figura 5). 


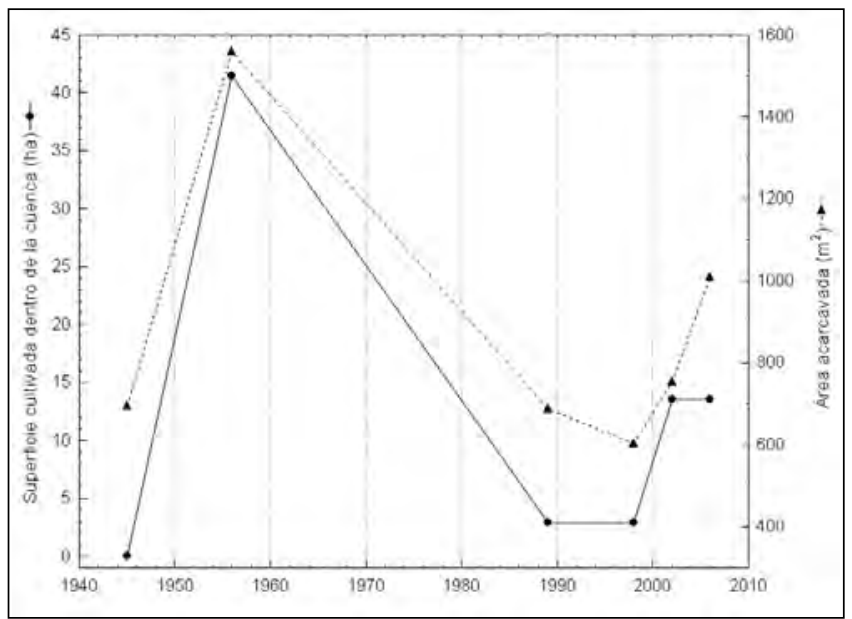

Figura 4. Evolución comparada de la superficie cultivada y el área acarcavada en la cuenca Parapuños desde 1945 hasta 2006.

Tabla 5. Elementos registrados en cada ortofotografía

\begin{tabular}{|l|c|c|c|c|}
\hline Fecha & $\begin{array}{c}\text { Longitud de las líneas } \\
\text { de incisión (m) }\end{array}$ & $\begin{array}{c}\text { Área acarcavada } \\
\mathbf{( m}^{\mathbf{2}} \mathbf{)}\end{array}$ & $\begin{array}{c}\mathbf{N}^{\mathbf{o}} \mathbf{d e} \\
\text { cabeceras }\end{array}$ & $\begin{array}{c}\text { Superficie } \\
\text { cultivada (ha) }\end{array}$ \\
\hline $28-30 / 09 / 1945$ & 607,84 & 695,22 & 5 & 0,00 \\
$17 / 04 / 1956$ & 872,18 & $1.559,58$ & 6 & 41,47 \\
$08 / 1989$ & 835,08 & 688,07 & 6 & 2,85 \\
$02 / 1998$ & 701,28 & 604,64 & 10 & 2,85 \\
$19 / 06 / 2002$ & 933,59 & 753,75 & 22 & 13,50 \\
$26-29 / 04 / 2006$ & $1.003,5$ & $1.009,32$ & 23 & 13,50 \\
\hline
\end{tabular}

Tabla 6. Evolución de la superficie acarcavada y precipitación para cada período (en las columnas 5 y 6 el aumento del acarcavamiento se considera positivo y la disminución del área acarcavada negativa

\begin{tabular}{|l|c|c|c|c|c|}
\hline Período & $\begin{array}{c}\text { Duración } \\
\text { (años) }\end{array}$ & $\begin{array}{c}\text { Pp total } \\
\mathbf{( m m})\end{array}$ & $\begin{array}{c}\text { Pp media } \\
(\mathbf{m m})\end{array}$ & $\begin{array}{c}\text { Evolución del área } \\
\text { acarcavada }\left(\mathbf{m}^{\mathbf{2}}\right)\end{array}$ & $\begin{array}{c}\text { Evolución del área } \\
\text { acarcavada }\left(\mathbf{m}^{2} / \mathbf{a n n o}\right)\end{array}$ \\
\hline PI (1945-56) & 10,52 & 4.868 & 463 & 864 & 82,2 \\
PII (1956-89) & 33,25 & 16.915 & 509 & -871 & $-26,2$ \\
PIII (1989-98) & 8,75 & 4.478 & 512 & -83 & $-9,5$ \\
PIV (1998-2002) & 4,33 & 1.147 & 265 & 149 & 34,4 \\
PV (2002-06) & 3,75 & 1.814 & 484 & 256 & 68,2 \\
\hline
\end{tabular}




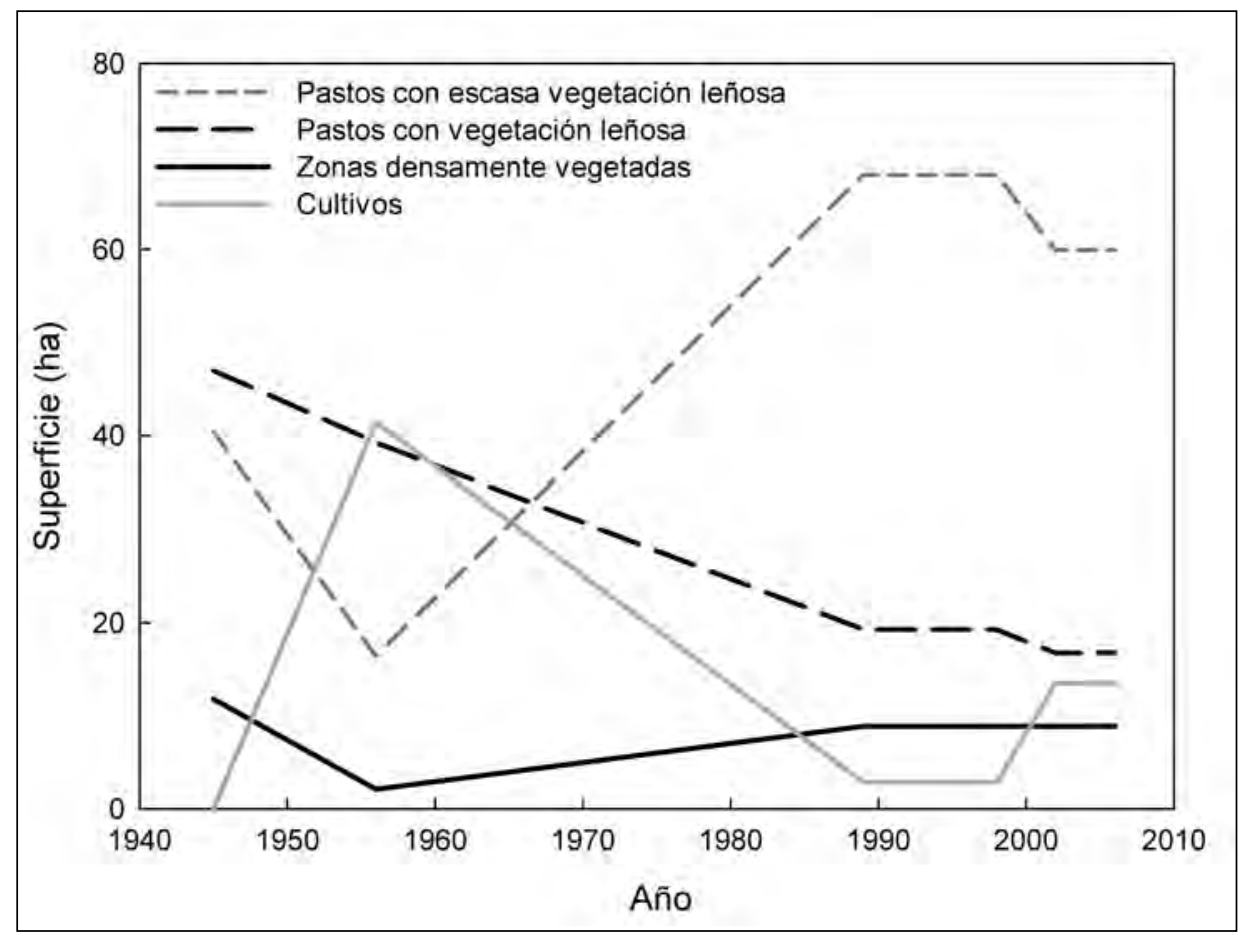

Figura 5. Evolución de diferentes tipos de agrupaciones de uso del suelo y cubierta vegetal. Los 33 usos del suelo incluidos en el catálogo de usos se han agrupado en 4 grandes grupos de usos o cubiertas.

Posteriormente, en las ortofotografías de 2002 y 2006 se observa un avance del acarcavamiento a razón de 34,4 $\mathrm{m}^{2}$ año-1 (PIV) y 68,2 $\mathrm{m}^{2}$ año-1 (PV). Durante este período (1998-2006), la superficie cultivada dentro de la cuenca aumenta de nuevo. Este incremento se debe a la introducción de un cultivo cuyo fin es la atracción de diversas especies de aves para caza menor (tórtolas, palomas, etc.). Se trata de una zona bastante llana, donde domina la infiltración frente a la generación de escorrentía y que además no se encuentra claramente conectada con gran parte de la cárcava. Por este motivo, dudamos que dicho cultivo tenga una relación directa con la extensión de la cárcava durante este período. Este aumento de la superficie afectada por la cárcava desde 1998 coincide con un incremento notable en la carga ganadera de la explotación que pasó de soportar entre 500-600 ovejas a finales de los 90 a unas 1.800 ovejas y 140 cerdos a partir del 2000.

En términos generales, la cubierta vegetal experimenta un descenso desde 1945 hasta 2006. Esta pérdida está relacionada con la puesta en cultivo de gran parte de la superficie dentro de la cuenca y la pérdida de pastizales con vegetación leñosa (Figura 5). Después de 1956, cuando los cultivos son abandonados, se transforman en pastizales con poca vegetación leñosa. La densidad arbórea experimenta un elevado descenso durante 
el período de estudio, pasando de 28,85 pies ha-1 en 1945 a 21,20 pies ha-1 en 2006. Si bien es cierto, que durante los últimos años se ha constatado una estabilización de la densidad arbórea (con 21,1 pies ha ${ }^{-1}$ en 2002 y 21,2 pies ha $^{-1}$ en 2006).

Finalmente se analizó la influencia de la precipitación acaecida durante cada período sobre el desarrollo de la cárcava. No se encontró ninguna relación significativa entre ambas. Las correlaciones entre la evolución de la superficie acarcavada y las cantidades totales de precipitación en cada período no arrojaron coeficientes significativos. Tampoco lo hicieron el número de años húmedos por período (con precipitación superior al percentil 0,8), ni el número de años húmedos seguidos por período. En la Tabla pueden observarse los valores de precipitación obtenidos para cada período.

\section{Discusión}

Los resultados de la monitorización de la cárcava desde 2001 hasta 2006 demuestran la estrecha relación de la precipitación y el caudal con los procesos de erosión en cárcava, especialmente con la existencia de caudales elevados. Se trata de tan sólo 9 períodos de observación, sin embargo las relaciones obtenidas son consistentes, y vienen a mejorar el conocimiento del proceso de acarcavamiento y su relación con el caudal y la precipitación. La disponibilidad de información similar en la bibliografía es escasa (Crouch, 1990; Thomas et al., 2004), especialmente en ambientes con una alta variabilidad anual y estacional de las precipitaciones. La estacionalidad de las pérdidas, con valores elevados durante otoño y principio de invierno, se relaciona con los eventos de caudal de mayor magnitud y por tanto mayor capacidad erosiva. Asimismo, la disponibilidad de sedimentos es mayor al inicio del año hidrológico. Durante la segunda mitad del año hidrológico, las pérdidas observadas parecen relacionarse con el contenido de agua del suelo y por tanto con la frecuencia de los eventos de precipitación, dando lugar a movimientos de material dentro de la cárcava (colapso de las márgenes y retroceso de la cabecera).

Dada la frecuencia del registro de datos de erosión en cárcava para este período, fue imposible realizar un análisis a escala de evento. Tampoco se observaron eventos de características excepcionales durante el período de estudio, más bien todo lo contrario, algunos períodos resultaron especialmente secos. Bajo condiciones ambientales similares a Parapuños, Schnabel et al. (1999) observaron enormes pérdidas ocasionadas por un evento con un período de retorno de 200 años en una cárcava permanente de fondo de valle.

Los resultados del registro del área acarcavada para el período 1945-2006 muestran que el cambio en la escala temporal de análisis del fenómeno puede enmascarar el papel de determinados factores (precipitación, caudal). Al mismo tiempo, la ampliación del marco temporal de trabajo, facilita la incorporación de factores cuya influencia es difícilmente evaluable a partir de estudios de varios años (1-10 años).

La falta de relación entre los datos de precipitación y el desarrollo de la cárcava a través del análisis de las ortofotografías, puede explicarse bajo dos premisas: i) el bajo 
número de observaciones de que se dispone (6 fotografías aéreas; 5 períodos) y/o ii) la imposibilidad de las cantidades de precipitación total anual para reflejar las características de la precipitación que determinan la iniciación y avance de los procesos de acarcavamiento, como las características individuales de cada evento (intensidad y duración) y la distribución anual de los mismos. Stocking (1980) ya señaló la dificultad de analizar la influencia de la precipitación sobre el acarcavamiento utilizando datos de baja resolución temporal que pueden ocultar el papel de los eventos individuales.

El incremento del área acarcavada desde 1945 hasta 1956 coincide con la puesta en cultivo de casi la mitad de la superficie de la cuenca. Este aumento del acarcavamiento se relaciona con un incremento en la producción de escorrentía superficial y un descenso en la cohesión de la parte superficial del suelo. La relación entre el desarrollo de cárcavas y la deforestación originada por la puesta en cultivo de grandes superficies ya ha sido documentada por algunos autores (Sirviö y Rebeiro-Hargrave, 2004).

La disminución de la superficie afectada por acarcavamiento que se produce desde 1956 hasta 1989 está relacionada con el abandono de casi la totalidad de los cultivos. Se produce una revegetación de la cuenca, especialmente de las vaguadas, dada su situación topográfica. También aparece vegetación en las secciones del cauce y sus paredes verticales se convierten en márgenes de pendientes suaves y estables, reduciéndose así la superficie acarcavada que puede delimitarse a partir de las ortofotografías. En definitiva, tiene lugar una disminución de la actividad del proceso de acarcavamiento consecuencia general del aumento de la resistencia del suelo frente a la erosión (ya que las prácticas de laboreo desaparecen) y una disminución de la escorrentía superficial generada así como de su velocidad y por tanto de su capacidad erosiva, debido a la revegetación de la cuenca. La naturaleza cíclica de los procesos de acarcavamiento, es de sobra conocida (Bradford y Piest, 1980). De manera que el volumen de una cárcava puede decrecer a partir de un determinado momento, relacionándose este hecho con dinámicas de sedimentación y revegetación del cauce (Nachtergaele et al., 2002).

La reactivación del acarcavamiento durante los últimos años está relacionada con un notable aumento de la carga ganadera. La presencia de elevadas cargas origina un descenso en la cubierta vegetal y provoca una compactación de la parte superficial de suelo, disminuyendo su capacidad de infiltración y aumentando por tanto la producción de escorrentía. Este hecho coincide con las tendencias regionales constatadas por otros autores (Lavado Contador et al., 2004; Plieninger, 2006) y que muestran un aumento notable de la cabaña ganadera como consecuencia del ingreso de España en la Unión Europea y de la adopción de la Política Agraria Común que basará sus subvenciones en el número de cabezas de ganado potenciando el sobrepastoreo (Lyrintzis, 1996). El sobrepastoreo, ha sido señalado en algunos trabajos como causa principal de la aparición y desarrollo de cárcavas en zonas de pastoreo de Ecuador (Podwojewski et al., 2002), de Nueva Zelanda (Gómez et al., 2003), de Etiopía (Nyssen et al., 2004), en la isla chilena de Rapa Nui (Mieth y Bork, 2005) y también en zonas agrarias y de pastoreo de Italia (Zucca et al., 2006). Actualmente el sobrepastoreo parece el principal problema para la conservación de los suelos de algunos espacios adehesados, especialmente en las vaguadas. 


\section{Conclusiones}

Las tasas de erosión registradas son muy variables, muestran cierta estacionalidad y se relacionan con la disponibilidad de sedimentos, los caudales elevados y la humedad antecedente. Cuando se amplia la óptica temporal de análisis, factores como el uso del suelo o la presión antrópica a la que se somete éste, adquieren especial relevancia. La superficie dedicada a cultivos así como la presencia de elevadas cargas ganaderas está relacionada con la evolución de la cárcava de Parapuños. Actualmente el sobrepastoreo parece el principal causante del desarrollo de la cárcava. A tenor de los resultados obtenidos y del desconocimiento de algunos aspectos de los procesos de aparición y desarrollo de cárcavas se hace necesario el impulso de trabajos e investigaciones en esta línea, con el fin de profundizar en el conocimiento del proceso y así poder ofrecer al agricultor herramientas y medidas de prevención y control.

\section{Agradecimientos}

Financiado por el Ministerio de Educación y Ciencias, proyectos AMB92-0580, AMB95-0986-C02-02, HID98-1056-C02-02, REN2001-2268-C02-02, CGL200404919-C02-02 y Ministerio de Medio Ambiente, RESEL.

\section{Referencias bibliográficas}

BRADFORD, J.M., PIEST, R.F. (1980). Erosional development of valley-bottom gullies in the Upper Midwestern United States. En: Thresholds in Geomorphology (Coates, D., Vitek, J.D., Eds.) London, Allen \& Unwin.

CROUCH R.J. (1990). Rates and mechanisms of discontinuous gully erosion in a redbrown earth catchment, New South Wales, Australia. Earth Surface Processes and Landforms, 15, 277-282.

Chaplot, V., Coadou Le Brozec, E., Silvera, N., Valentin, C. (2005). Spatial and temporal assessment of linear erosion in catchments under sloping lands of northern Laos. Catena, 63, 167-184.

FAULKNER, H. (1995). Gully erosion associated with the expansion of unterraced almond cultivation in the coastal Sierra de Lujar, S. Spain. Land Degradation \& Rehabilitation, 9, 179-200.

Gómez, B., Banbury, K., Marden, M., Trustrum, N.A., Peackock, D.H., Hoskin, P.J. (2003). Gully erosion and sediment production, Te Weraroa Stream, New Zealand. Water Resources Research, 39.

HARVEY, A.M. (1996). Holocene hillslope gully systems in the Howgill Fells, Cumbria. En Advances in Hillslope Processes (Anderson, M. G., Brooks, S. M., Eds.). Wiley 
KASAI, M. (2006). Channel processes following land use changes in a degrading steep headwater stream in North Island, New Zealand. Geomorphology, 81, 421-439.

LAVAdo CONTADOR, J.F., SCHNABEL, S., TRENAdo ORdóÑEZ, R. (2004). Comparison of recent land use and land cover changes in two dehesa agrosilvopastoral landuse systems SW Spain. En: Sustainability of agrosilvopastoral Systems (Dehesas, Montados). (Schnabel, S. y Ferreira, A., Eds.) Advances in Geoecology, 37, Catena Verlag.

LYRINTZIS, G.A. (1996). Human impact trend in Crete: the case of Psilorites Mountain. Environmental Conservation, 23, 140-148.

MARTíNEZ-CASASNOVAS, J.A., SÁNCHEZ-BOSCH, I. (2000). Impact assessment of changes in land use/conservation practices on soil erosion in the Penedès-Anoia vineyard region (NE Spain). Soil \& Tillage Research, 57, 101-106.

MiETH, A., BORK, H.R. (2005). History, origin and extent of soil erosion on Easter Island (Rapa Nui). Catena, 63, 244-260.

Nachtergaele, J., Poesen, J., Oostwoud Wijdenes, D., VAndeKerckhove, L. (2002). Medium-term evolution of a gully developed in a loess derived soil. Geomorphology, 46, 223-239.

Nyssen, J., Poesen, J., Moeyersons, J., Deckers, J., Mitiku, H., Lang, A. (2004). Human impact on the environment in the Ethiopian and Eritrean highlands: a state of the art. Earth Science Reviews, 64, 273-320.

Parkner, T., Page, M.J., Marutami, T., Trustrum, N.A. (2006). Development and controlling factors of gullies and gully complexes, East coast, New Zealand. Earth Surface Processes and Landforms, 31, 187-199.

Plieninger, T. (2006). Las dehesas de la penillanura cacereña: origen y evolución de un paisaje cultural, Servicio de Publicaciones de la Universidad de Extremadura.

Plieninger, T., HARALD, S. (2006). Elementos estructurales del paisaje adehesado tradicional en Monroy y Torrejón el Rubio (Cáceres y su importancia para la conservación de la naturaleza y el desarrollo rural. Revista de estudios extremeños, LXII, 441-483.

Podwojewski, P., Poulenard, J., Zambrana, T., Hofstede, R. (2002). Overgrazing effects on vegetation cover and properties of volcanic ash soil in the paramo of Llangahua and La Esperanza (Tungurahua, Ecuador). Soil Use and Management, $18,45-55$.

Prosser, I.P., WinChESTER, J. (1996). History and processes of gully initiation and development in eastern Australia. Zeitschrift für Geomorphologie, 105, 91-109.

SCHNABEL, S. (1997). Soil erosion and runoff production in a small watershed under silvopastoral landuse (dehesas) in Extremadura, Spain, Logroño, Geoforma Ediciones. 
Schnabel, S., Gómez Amelia, D., Ceballos, A. (1999). Extreme events and gully erosion. En Proceedings of the International Seminar on Land Degradation and Desertification. IGU, Lisbon.

SiRviö, T., REBEIRO-HARGRAVE, A. (2004). Hazardous foothills: An overview of gully erosion in Taita Hills. Expedition reports of the Department of Geography of Helsinki University, 40, 79-86.

STOCKING, M.A. (1980). Examination of the factors controlling gully growth. En: Assesment of erosion (Boodt, D., Gabriels, D., Eds.), Willey, Chichester, pp. 505-521.

ThOMAs, J.T., IVERSON, N.R., BURKART, M.R., KRAMER, L.A. (2004). Long term growth of a valley-bottom gully, wester Iowa. Earth Surface Processes and Landforms, 29, 995-1009.

Zucca, C., Cannu, A., Della Peruta, R. (2006). Effects of land use and landscape on spatial distribution and morphological features of gullies in an agropastoral area in Sardinia (Italy). Catena, 68, 87-95. 